Relations industrielles

Industrial Relations

\title{
Sarfati, Hedva, Flexibilité et créations d'emplois : un défi pour le dialogue social en Europe
}

\section{André Beaucage}

Volume 54, numéro 4, 1999

URI : https://id.erudit.org/iderudit/051282ar

DOI : https://doi.org/10.7202/051282ar

Aller au sommaire du numéro

Éditeur(s)

Département des relations industrielles de l'Université Laval

ISSN

0034-379X (imprimé)

1703-8138 (numérique)

Découvrir la revue

Citer ce compte rendu

Beaucage, A. (1999). Compte rendu de [Sarfati, Hedva, Flexibilité et créations d'emplois : un défi pour le dialogue social en Europe]. Relations industrielles / Industrial Relations, 54(4), 834-836. https://doi.org/10.7202/051282ar

Tous droits réservés @ C Département des relations industrielles de l'Universite Laval, 1999
Ce document est protégé par la loi sur le droit d'auteur. L’utilisation des services d'Érudit (y compris la reproduction) est assujettie à sa politique d'utilisation que vous pouvez consulter en ligne.

https://apropos.erudit.org/fr/usagers/politique-dutilisation/ 
ouvrage, devrait ėtre conçue davantage comme le résultat d'un processus coopératif plutôt que d'une rencontre entre

DIANE BELLEMARF

offreurs et demandeurs.

Département de ressources humaines

et d'organisation

UQAM

\section{Flexibilité et créations d'emplois : un défi pour le dialogue social en Europe} par Hedva SARFATI, Paris et Montréal : l'Harmattan, 1999, 229 p., ISBN 2-7384$7722-4$

La hausse tendancielle des taux de chômage dans plusieurs pays depuis les années 1970 , ou leur stagnation à des niveaux jugés trop élevés, ainsi que la crainte de nouvelles flambées inflationnistes, ont amené une remise en question des politiques macro-économiques traditionnelles et favorisé un retour en force du credo néoclassique dans l'efficacité du libre jeu des forces du marché. Dans un contexte de concurrence accrue et de plus en plus internationale, la solution au chômage doit désormais passer par l'élimination des contraintes imposées à la variation des prix et à la mobilité des produits et de leurs facteurs de production. Dans le monde du travail, la recette se traduit par une plus grande capacité d'adaptation que doivent obtenir les entreprises dans l'utilisation et la rémunération de leur main-d'œuvre. Les stratégies de flexibilité du marché du travail devraient permettre à ces entreprises d'être plus compétitives et ainsi protéger, voire augmenter, les emplois. Ces stratégies visent une diminution des coûts de main-d'œuvre par une réduction des salaires et de certains avantages sociaux (flexibilités salariale et numérique) ou par une utilisation plus optimale du temps de travail (flexibilité numérique). La diminution des coûts de main-d'œuvre peut également être obtenue par une plus grande efficacité dans l'organisation de la production et du travail (flexibilité fonctionnelle). Cette dernière stratégie a reçu un accueil plus favorable des organisations syndicales dont plusieurs voyaient dans la réduction de la semaine de travail une des solutions au chômage endémique.

L'ouvrage d'Hedva Sarfati, politologue et ex-directrice du Département des re- lations professionnelles et de l'administration du travail au Bureau international du travail, questionne les mérites des stratégies de flexibilité du marché du travail pour une compétitivité accrue des entreprises et pour la protection et le développement de l'emploi. Son étude porte sur l'expérience de onze pays européens : l'Allemagne, l'Autriche, la Belgique, l'Espagne, la Finlande, la France, l'Irlande, l'Italie, les Pays-Bas, le Portugal et le Royaume-Uni. "Elle tente de démontrer que, dans cette difficile quête d'un équilibre entre flexibilité et sécurité, de meilleurs résultats ont pu être obtenus, pour les salariés comme pour les entreprises, dans les pays où le dialogue social existe ou a pu être mis en place, c'est-à-dire là où prévaut un climat de confiance entre les partenaires sociaux * (p. 7). Pour atteindre cet objectif, l'auteure s'est appuyée sur une revue des mesures législatives et des conventions collectives négociées à différents niveaux (national, sectoriel et d'entreprise) pour promouvoir l'emploi et réduire le chômage dans les pays étudiés.

L'étude comprend trois parties de deux chapitres chacune. Après une préface et une introduction qui se répètent sur certains aspects, la première partie de l'ouvrage ambitionne de retracer l'évolution du débat sur la flexibilité du marché du travail. Dans un premier chapitre, Hedva Sarfati situe le contexte de ce débat en s'appuyant sur le contenu de certaines études de l'OCDE et de la Commission européenne sur l'emploi et sur une enquête d'opinion réalisée par la Fondation européenne pour l'amélioration des conditions de vie au travail auprès des chefs d'entreprise sur la participation des salariés aux décisions. Elle 
arrive à la conclusion qu'il est nécessaire " de faire la part des choses, dans le débat sur la flexibilité entre les facteurs économiques et les facteurs sociaux, lorsque l'on s'efforce de résoudre les problèmes de la compétitivité de l'entreprise et de la croissance de l'emploi " (p. 36). Le chapitre 2 est une longue présentation (le tiers de l'ouvrage) des attitudes et des actions des partenaires sociaux des pays visés face au problème de l'emploi. On y présente successivement l'évolution de la position des gouvernements des onze pays, celle des syndicats de quatre pays et des employeurs et associations d'employeurs de cinq pays. Il est très difficile de faire une synthèse des nombreuses observations de ce chapitre et d'en dégager les lignes directrices. L'auteure ne s'y aventure pas non plus.

La deuxième partie de l'ouvrage est consacrée au dialogue social entre les partenaires sociaux. Le chapitre 3 traite d'abord des contraintes sur ce dialogue social à l'échelon national ou sectoriel qui découlent de la faiblesse des partenaires sociaux (problème de représentativité des syndicats et des associations d'employeurs, affaiblissement de l'autonomie politique des gouvernements), de l'importance grandissante des entreprises multinationales et de l'accélération des changements technologiques, de la réorganisation du travail, de la mondialisation de l'économie et du processus d'intégration européenne. Le chapitre 4, qui doit être lu en revenant régulièrement sur les observations du chapitre 2 , nous présente la diversité des accords pour la protection de l'emploi négociés aux niveaux national, sectoriel ou de l'entreprise. L'auteure en dégage cinq grands constats: (i) Les négociations collectives nationales ou sectorielles se sont transformées, mais ont survécu. (ii) La négociation collective a contribué positivement à l'élaboration des politiques de lutte contre le chômage. (iii) Différents niveaux de négociation coexistent dans un même pays, mais portent sur des questions différentes. (iv) La décen- tralisation de la négociation vers l'entreprise est évidente, mais on ne saurait surestimer son impact global. (v) Enfin, il y a complémentarité entre la législation et la négociation collective pour la protection et la promotion de l'emploi.

Dans la dernière partie de son livre, Hedva Sarfati tente d'abord, au chapitre 5 , de situer le dialogue social et la promotion de l'emploi dans le contexte de l'intégration européenne. Elle souligne les limites et le potentiel d'une concertation à ce niveau à partir de la lecture qui est faite des traités de Maastricht (1991) et d'Amsterdam (1997), de certains accords conjoints négociés au niveau européen (sur le congé parental, la discrimination des travailleurs à temps partiel et le travail à durée déterminée), et au niveau sectoriel (agriculture, commerce et distribution), et de l'arrivée au pouvoir de onze gouvernements à majorité socialiste. Finalement, dans le chapitre 6 qui complète l'ouvrage, elle propose quelques leçons des expériences en matière de dialogue social sur la flexibilité et l'emploi, tout en réfutant certaines idées reçues. Ainsi, la flexibilité du marché du travail aurait davantage favorisé le développement de l'emploi atypique et réduit la protection sociale des travailleurs que provoqué une diminution du chômage. De même, l'efficacité de la réduction des heures de travail pour augmenter l'emploi ne serait pas démontrée, tout comme l'impact négatif du salaire minimum et de la libéralisation des échanges internationaux sur l'emploi. De nombreux autres facteurs (productivité, innovation, formation, qualité des produits, etc.) doivent être considérés pour expliquer l'évolution de l'emploi et pour comprendre l'effort d'adaptation qui est exigé des entreprises qui veulent demeurer compétitives et protéger leurs emplois. Les différents niveaux du dialogue entre les partenaires sociaux sont présentés comme des moyens privilégiés pour faciliter cet effort d'adaptation.

Cette brève présentation du contenu de l'ouvrage de Madame Sarfati ne peut 
rendre justice à la variété des informations et à la richesse des observations qu'il renferme sur la façon de résoudre, par les partenaires sociaux européens, le dilemme de la protection de l'emploi (sécuritê) dans une économie aux exigences de productivité et d'efficacité (flexibilité) sans cesse relevées. Le lecteur moins familier avec les expériences européennes pour la promotion de l'emploi y trouvera plusieurs présentations éclairantes et des observations fort stimulantes. Il sera cependant dérouté par l'organisation de l'ouvrage qui rend la synthèse du propos fort difficile. L'auteure ne propose pas à la fin de chaque chapitre les lignes directrices qu'il faudrait retenir des nombreuses observations qui y sont faites. Pour ajouter à la difficulté, dans plusieurs chapitres, on revient sur les expériences des pays (pas toujours les mêmes?) dont on présente et discute des aspects différents mais complémentaires du dialogue social entre les partenaires. Pour avoir une compréhension plus complète de l'expérience, il faut retoumer fréquemment au contenu des autres chapitres du livre (souvent à la suggestion de l'auteure). Enfin, Edva Sarfati ne présente pas le cadre conceptuel qui serait sous-jacent à sa démarche. En réalité, l'ouvrage se limite le plus souvent à décrire et à commenter de nombreux cas de négociation ou de législation pour la protection de l'emploi. La démonstration des succès de ces expériences de dialogue social ou des insuccès des stratégies de flexibilité du marché du travail est malheureusement loin d'être toujours convaincante. Elle s'appuie d'ailleurs (chapitre 6) plus souvent sur les résultats d'autres recherches que sur ceux de l'étude qui nous a été soumise.

ANDRÉ BEAUCAGE

Département de relations industrielles Université du Québec à Hull

\section{Strikes and Solidarity : Coalfield Conflict in Britain 1889-1966}

by Roy A. CHURCH and Quentin OUTRAM, Cambridge : Cambridge University

Press, 1998, 314 p., ISBN 0-521-55460-8.

This is an impressive and challenging book which should be of interest not only to coalmining labour historians but to industrial relations scholars more generally and to many industrial sociologists. Roy Church and Quentin Outram argue that while there is now a vast literature relating to coalfield conflict in Britain, serious deficiencies persist in our knowledge of the historical pattern of coalmining strike activity which have obscured understanding of the militancy for which British coalminers have been famed. In setting out to provide a more complete account, they focus on local and domestic strikes which, in terms of frequency and prevalence, constituted the industry's "ordinary" strikes; the large-scale county, regional and national disputes given prominence in much of the literature were important but rare in their incidence. The analysis spans a time-period defined largely by the availability of the main primary source used, that is the official statistical record of strikes and lockouts compiled by the Board of Trade from 1901. As Church and Outram emphasize, it is the "unique degree of disaggregation" of the data contained in these manuscript ledgers that has enabled a much more detailed examination of the forms and variations of strike activity than has been attempted hitherto. Their study aims to explain the causes of strikes and the temporal, spatial and inter-colliery differences in strike activity identified. More ambitiously, it seeks to contribute to the development of a "new labour studies agenda" (p. xvii). In relation to this broader intellectual project, Church and Outram highlight the potential of their methodology which combines "traditional historical research methods and quantitative analysis with 